\title{
Building a predictive model for PHB production from glycerol
}

\section{DOI:}

10.1016/j.bej.2016.04.016

\section{Document Version}

Accepted author manuscript

Link to publication record in Manchester Research Explorer

\section{Citation for published version (APA):}

Perez rivero, C., Sun, C., Theodoropoulos, K., \& Webb, C. (2016). Building a predictive model for PHB production from glycerol. Biochemical Engineering Journal, 116, 113-121. https://doi.org/10.1016/j.bej.2016.04.016

\section{Published in:}

Biochemical Engineering Journal

\section{Citing this paper}

Please note that where the full-text provided on Manchester Research Explorer is the Author Accepted Manuscript or Proof version this may differ from the final Published version. If citing, it is advised that you check and use the publisher's definitive version.

\section{General rights}

Copyright and moral rights for the publications made accessible in the Research Explorer are retained by the authors and/or other copyright owners and it is a condition of accessing publications that users recognise and abide by the legal requirements associated with these rights.

\section{Takedown policy}

If you believe that this document breaches copyright please refer to the University of Manchester's Takedown Procedures [http://man.ac.uk/04Y6Bo] or contact uml.scholarlycommunications@manchester.ac.uk providing relevant details, so we can investigate your claim.

\section{OPEN ACCESS}




\title{
Building a predictive model for PHB production from glycerol
}

\author{
Cristina Pérez Rivero, Chenhao Sun, Constantinos Theodoropoulos and Colin Webb*
}

School of Chemical Engineering and Analytical Science, University of Manchester, M13 9PL, UK

\begin{abstract}
Poly-3-hydroxybutyrate (PHB) is a biodegradable biopolyester with plastic like properties, which on its own or as part of a heteropolymer, finds application in everyday products, competing directly with fossil fuel based plastics in terms of physical and mechanical properties. In nature, PHB is produced as an energy reservoir for the host cell, when environmental conditions limit growth. It is this inherent condition for PHB synthesis (i.e. an environment unsuitable for growth) that challenges design of conventional batch production systems. Balance between growth (driven by nitrogen availability) and PHB production (enhanced by an excess of carbon) is the critical aspect for consideration in such designs. However, selecting the best operating conditions is not obvious for this system and so a systematic approach has been used in this paper, utilising simulations based on a purpose built model to supplement experimental studies.

The interaction between the carbon and nitrogen sources (glycerol and ammonium sulphate respectively) was carefully evaluated and incorporated into a low-structured model able to describe the dynamics of substrate consumption and product accumulation during C.necator DSM 545 cultivation at small scale. The kinetic parameters thus determined have been assumed to be constant, fixed accordingly, and the model used to predict the fermentation profiles for different operating conditions. Results showed good agreement with experimental data, supporting the efficacy of this approach. The dual utilization of multiple substrates suggests there is a system capacity to which both growth and PHB production contribute and that sets the maximum total biomass concentration. A logistic type term added to both growth and product rate equations enabled the effective decoupling of cell proliferation and PHB accumulation for a wide range of scenarios. In this way, the combination of predictive modelling and experimental verification potentially reduces, by a significant amount, the number of experiments required to establish operational targets such as specific growth rate and productivity as well as identifying often sought criteria such as optimum $\mathrm{C}: \mathrm{N}$ ratio.
\end{abstract}

Keywords: Biorefinery; Intracellular polymer; Predictive Modelling; Growth Kinetics; Production Kinetics; Dual substrate model

*Corresponding author: Colin Webb: colin.webb@manchester.ac.uk

Telephone number: +441613064379

Short running title: Building a predictive model for $P H B$ production 


\section{List of symbols:}

$G l y$
$k_{d}$
$k_{G}$
$k_{I G}$
$k_{I N}$
$k_{I N P}$
$k_{N}$
$k_{P}$
$K_{x}$
$n$
$N$
$S$
$Y{ }_{P H B} / G l y$
$Y x_{R} / N$
$Y x_{R} / G l y$
$\beta$
$\mu_{m}$
$\mu_{p}$
$\mu_{x_{R}}$

Glycerol concentration (g/L)

Apparent dissociation constant $(\mathrm{g} / \mathrm{L})$

Half saturation constant for glycerol (g/L)

Glycerol inhibition constant $(\mathrm{g} / \mathrm{L})$

Nitrogen inhibition constant (g/L)

Inhibition constant for PHB production $(\mathrm{g} / \mathrm{L})$

Half saturation constant for nitrogen $(\mathrm{g} / \mathrm{L})$

Half saturation constant for PHB (g/L)

Carrying capacity $(\mathrm{g} / \mathrm{L})$

Hill coefficient (-)

Nitrogen concentration $(\mathrm{g} / \mathrm{L})$

Substrate concentration $(\mathrm{g} / \mathrm{L})$

Yield of PHB based on glycerol $(\mathrm{g} / \mathrm{g})$

Yield of non-PHB biomass based on nitrogen $(\mathrm{g} / \mathrm{g})$

Yield of non-PHB biomass based on glycerol $(\mathrm{g} / \mathrm{g})$

Non growth associated specific rate constant $(1 / \mathrm{h})$

Maximum specific growth rate $(1 / \mathrm{h})$

Specific production rate $(1 / \mathrm{h})$

Specific growth rate $(1 / \mathrm{h})$

\section{Introduction}

Interest in poly-3-hydroxybutyrate rose due to different reasons since its discovery in 1927 . For example, in the 1970s, matching the peak in oil prices, substitutes to petrol-derived plastics seemed necessary to cover the polymer demand. More recently, environmental awareness has motivated the research into non-chemical routes for producing bioplastics, particularly from renewable resources to be used as feedstocks for microbial cultivation [1]. Through such products, the disposal problems at the end of life of plastics are set to disappear. PHB is biodegradable and the carbon dioxide released when it degrades is balanced in part by that used for the growth of the biomass, decreasing net greenhouse gas emissions and solids wastes sent to landfills [2]. Besides, the independence from fossil fuel sources remains a key factor for pushing research and development in this area.

The physical characteristics of PHB are similar to those of polypropylene, making it a suitable candidate to replace traditional plastics in applications such as food packaging films, biodegradable carriers for medicines and insecticides, disposable cosmetic products, surgical devices and starting compounds for chiral substances [3]. Nevertheless, technology lags behind that necessary to reach all these markets. Expensive raw materials, insufficient fermentation optimization and poor product recovery are highlighted as the most important reasons for the scarce industrial implementation [4]. To deal with these issues, more efficient microorganisms that utilize low cost substrates together with a better control of the production process need to be established.

Though many bacteria have been isolated and characterised as natural PHB producers, with Azohydromonas lata (formerly known as Alcaligenes latus) and Azotobacter and Pseudomonas species as some of the most studied, there is no real competitor to Cupriavidus necator (formerly Alcaligenes eutrophus, Ralstonia eutropha) as an industrial strain. C. necator can utilize many different organic or inorganic carbon sources for accumulating large amounts of PHB (or its copolymers 
when feeding more than one carbon substrate). Special efforts have been put into using inexpensive renewable raw materials as fermentation feedstocks [5]. For example, under chemolithoautotrophic growth conditions, recycled mixtures of $\mathrm{H}_{2}$, $\mathrm{CO}_{2}$ and $\mathrm{O}_{2}$ have been used to produce $61.9 \mathrm{~g} / \mathrm{L}$ PHB in 40 hours, matching normal levels in a two stage fermentation of conventional sugars [6].

Mixed cultures have been explored to overcome two main limitations: the inability of $C$. necator to metabolize some common sugars and the additional substrates, besides glucose, required by some other bacteria in order to synthesize significant amounts of PHB [7]. When using microbial consortia, abundant sugars can be converted into organic acids by the latter species and those organic acids later used as substrate by $C$. necator to produce the biopolymer. In order to achieve higher productivities or, as commented, finding microorganisms that can readily utilize low-cost substrates, polyhydroxyalkoanate (PHA) biosynthesis genes from C. necator and Azohydromonas australica have been cloned into E. coli $[8]$. Although there are several studies on limiting the amount of oxygen as a strategy to promote PHB production during the accumulation stage, the desirable high growth rate of the $E$. coli cells requires a large oxygen supply. The alternative of employing anaerobic E. coli has the drawback of slower growth [9].

Glycerol is an example of those sustainable substrates that $C$. necator can readily utilize [10]. The current surplus of this polyalcohol, obtained as an intrinsic by-product from the transesterification reaction in biodiesel manufacture, has lowered the selling price and turned it into a waste rather than a coproduct. Its use as raw material in biological processes would contribute to the sustainable development of biorefineries for biodiesel production [11]. Methanol and sodium chloride have been found as the principal impurities in crude glycerol that affect the fermentation process by inhibiting cell growth [12, 13].

PHB production in $C$. necator cells is attributed to unbalanced nutritional conditions in which carbon is in excess over other key elements, such as nitrogen, phosphorus, oxygen or sulphur [14]. Under stress conditions, cells experience a switch from predominant biosynthesis of cellular components to production and accumulation of PHB, used as a carbon and energy storage system [15]. Many studies have explored the desirable conditions for yielding large amounts of PHB, e.g. the ratio of carbon to nitrogen concentrations and it is commonly accepted that the initial $\mathrm{C}: \mathrm{N}$ ratio determines the concentration of $\mathrm{PHB}$ that can be obtained through batch cultivation [16, 17]. However, such studies are most often empirical and the lack of a suitable bioreactor model limits the value of experimental data, prolonging the optimization process and reducing the general applicability of the results.

With essentially fixed yields (at least until more genetic information is released), and the substrate choice limited by the microorganism or by regional availability, reducing the cost gap between petroleum based plastics and bioplastics requires better fermentation process design, which brings the need for reliable kinetic and design models [18]. These can be of very different types, depending on the sort of information used for their development. Mechanistic models are based on the understanding of the behaviour of the system's components. They combine the kinetics of the bioprocess with mass balances and mass transfer phenomena. Some are developed from the review of earlier models with modifications being made to accommodate new findings. Thus, for example the differentiation between PHB and non-PHB biomass led to some authors referring to a catalytic fraction of the total biomass as the part that needs to be included in the Monod-type expression usually adopted for describing cell growth [19] or the fact that a severe shortage of nitrogen as well as an excess of it can prevent PHB formation [20]. The substrate inhibition model proposed by Luong in 1987 [21] and the logistic equation appear often to achieve a good fit for the experimental data [20, 22, 23].

Two-stage fermentation is the preferred configuration for the PHB production system, where the aim of the first stage is to achieve good growth; PHB production is then boosted during the second stage following exhaustion of, usually, a key nutrient. Although single stage continuous cultivation could result in a simpler and cheaper process, the low dilution rates 
necessary would reduce productivity and this mode of operation has only been used with certain microorganisms that exhibit high production rate during the exponential phase.

Good modelling provides a tool for design but only if constants can be transferred across systems and conditions. Lee $e t$ al. [24] searched for the optimal profiles for the feeding of carbon and nitrogen through different control strategies in order to maximize PHB production at the end of the fermentation. Macroscopic modelling together with information from metabolic fluxes was used by Katoh et al. [23] to refine the control strategy based on the addition of glucose (substrate for Lactobacillus delbrueckii) pulses whenever lactate (substrate for C.necator) concentration in the bioreactor fell below a setpoint. In contrast with past practices, Tohyama et al. [25] proposed a rate equation in which the specific growth rate was expressed as a function of dissolved oxygen concentration. More recently, Špoljarić et al. [26] developed a mathematical model to describe the conversion of substrates from biodiesel production (glycerol and fatty acid methyl esters) into poly[(R)-3-hydroxyalkanoate] by $C$. necator. Optimization of the fed-batch cultivation on glycerol, based on a low structured kinetic model, was then presented in a subsequent publication [27].

To achieve a more realistic representation of the cell machinery, cybernetic models add the action of internal regulatory controls, such as specific enzyme activities [28] while metabolic models consider the whole metabolic network. These provide in-sights into the internal workings of the cell and help identify bottlenecks and constraints, so that genetic engineering can be used to relieve them. In order to study the metabolic network of C. necator DSM 545, Lopar et al. [29] applied elementary flux modes and yield space analysis. Among the advantages of this class of models is the fact that they can describe transition stages caused by sudden changes in the system [30]. On the other hand, increased complexity, linked to a lack of detailed knowledge of the metabolism, limit their applicability. This limitation can be overcome to some extent through the use of artificial neural networks, which have been applied to model a great number of systems, including PHB synthesis [31]. They do not require a rigorous mathematical description but rather rely on an exhaustive training methodology, with solutions coming from a heuristic search. Hybrid models, which consider some level of understanding (mechanistic or cybernetic) in addition to empirical observation, are believed to represent the most sensible approach [32].

In this paper we describe a newly built predictive model for PHB production by $C$. necator DSM 545 using glycerol as the single carbon source. This is a relatively simple model that requires little computational effort when used for optimization purposes as it was intended for. Its robustness enables us to apply it throughout a wide range of conditions without any type of parameter tuning. The model building process has revealed some common features with other PHA systems (which can be exploited to promote general knowledge on biopolymer fermentations) but also some relevant insight of the particular system. The applied methodology has successfully demonstrated its usefulness for in silico optimization of the media composition, thus saving a large amount of trial and error experimentation.

Pure glycerol is the carbon source employed in this work, mimicking the biodiesel co-product stream. Considering that the main impurities of crude glycerol have previously been studied by our research group [13], using the pure form is still a good representation of the industrial waste and it provides a model system allowing us to generate fundamental knowledge without introducing too many disturbances from unknown chemical/biological interactions.

\section{Materials and methods}

\subsection{Strain and cultivation conditions}

Cupriavidus necator DSM 545 was obtained from the German Collection of Microorganisms and Cell Cultures (Deutsche Sammlung von Mikroorganismen und Zellkulture Gmb) in freeze dried form. The bacterial cells were reactivated in liquid media containing $5 \mathrm{~g} / \mathrm{L}$ peptone and $3 \mathrm{~g} / \mathrm{L}$ beef extract and then stored at $-30^{\circ} \mathrm{C}$ in vials prepared with the same media but 
supplemented with 20\% (v/v) glycerol. These vials served as inocula for sterilised preculture flasks containing (per litre): yeast extract, $1 \mathrm{~g} ;\left(\mathrm{NH}_{4}\right)_{2} \mathrm{SO}_{4}, 1 \mathrm{~g} ; \mathrm{KH}_{2} \mathrm{PO}_{4}, 1.5 \mathrm{~g} ; \mathrm{Na}_{2} \mathrm{HPO}_{4} \cdot 12 \mathrm{H}_{2} \mathrm{O}, 9 \mathrm{~g} ; \mathrm{MgSO}_{4} \cdot 7 \mathrm{H}_{2} \mathrm{O}, 0.2 \mathrm{~g} ; \mathrm{glycerol}, 30 \mathrm{~g}$ and $1 \mathrm{~mL}$ of trace element solution, as specified by $\mathrm{Kim}$ et al. [33]. The trace element solution was composed (per litre) by $\mathrm{FeSO}_{4} \cdot 7 \mathrm{H}_{2} \mathrm{O}_{\text {, }}$ $10 \mathrm{~g} ; \mathrm{ZnSO}_{4} \cdot 7 \mathrm{H}_{2} \mathrm{O}, 2.25 \mathrm{~g} ; \mathrm{CuSO}_{4} \cdot 5 \mathrm{H}_{2} \mathrm{O}, 1 \mathrm{~g} ; \mathrm{MnSO}_{4} \cdot 5 \mathrm{H}_{2} \mathrm{O}, 0.5 \mathrm{~g} ; \mathrm{CaCl}_{2} \cdot 2 \mathrm{H}_{2} \mathrm{O}, 2 \mathrm{~g} ;\left(\mathrm{NH}_{4}\right)_{6} \mathrm{Mo}_{7} \mathrm{O}_{24}, 0.1 \mathrm{~g} ; 35 \% \mathrm{HCl}, 10$ $\mathrm{mL}$. Erlenmeyer flasks of $500 \mathrm{~mL}$ capacity, containing $50 \mathrm{~mL}$ culture, were incubated at $30^{\circ} \mathrm{C}$ in orbital shakers at $220 \mathrm{rpm}$. Flasks were inoculated using preculture broth (5\% vol.) and cultivated under the same conditions. Yeast extract was just included in the preculture to promote a rapid growth but was absent from the cultivation medium due to its variable composition. The ammonium sulphate and glycerol concentration, single nitrogen and carbon source respectively, were varied in the different experiments conducted. All procedures were carried out aseptically where appropriate.

\subsection{Analytical methods}

Cell dry mass (CDM) determination: $1 \mathrm{~mL}$ culture was centrifuged in a $1.5 \mathrm{~mL}$ Eppendorf tube for 5 minutes at $9500 \mathrm{rcf}$. The supernatant was decanted into a new tube and stored in a freezer for subsequent glycerol and total nitrogen analysis. The Eppendorf tube containing the sediment was refilled with $1 \mathrm{~mL}$ distilled water for a second centrifugation. The supernatant from this was discarded and the pellet was suspended in distilled water and poured into a pre-weighed aluminium dish. The dish was placed in an oven at $60^{\circ} \mathrm{C}$ until constant weight in order to determine biomass concentration. CDM measurements were made in triplicate Optical density was correlated with growth and was used in the first hours of fermentation as an indicator of growth. For the measurement of optical density, the culture was diluted to measure absorbance at $600 \mathrm{~nm}$ in a UV-VIS Shimadzu spectrophotometer (Shimadzu, UV mini-1240, Japan).

HPLC Analysis of glycerol content: Glycerol consumption was determined using a High Performance Liquid Chromatograph equipped with a Hi-Plex $8 \mu \mathrm{m} 300 \times 7.7 \mathrm{~mm}$ column and coupled with and RI detector operating at $50^{\circ} \mathrm{C}$. The mobile phase consisted of an aqueous solution of $5 \mathrm{mmol}$ sulphuric acid. The temperature in the column was also set at $50^{\circ} \mathrm{C}$ and the flow rate and injection volume were $0.6 \mathrm{ml} / \mathrm{L}$ and $1 \mu \mathrm{L}$ respectively. Samples were diluted and filtered through $0.45 \mu \mathrm{m}$ prior to analysis.

Poly-3-hydroxybutyrate detection: The organic phase obtained from the extraction process described by Riis and Mai in 1988 was filtered and injected into a gas chromatograph (GC) Varian model CP-3800 assembled with autosampler Combi/Pal and coupled with a flame ionization detector (FID). The injection volume and the split ratio used were $1 \mu \mathrm{L}$ and 25:1 respectively. The temperature of the oven, where the Paraplot Q-HT column was placed, was $120^{\circ} \mathrm{C}$ at the time of injection and it was increased to $230^{\circ} \mathrm{C}$ after three minutes at a rate of $15^{\circ} \mathrm{C} / \mathrm{min}$. The sample was volatilized and passed through a slightly polar column using helium as carrier gas. A hydrogen / air flame oxidised organic molecules and ionized them. The signal produced when the ions hit the collector is proportional to the concentration of the substance analysed. Samples and standards were prepared following the same procedure described elsewhere in the literature [34]. Chemicals were obtained from Sigma and were of the highest grade available.

TN Analysis: Total dissolved nitrogen in the media was determined using a Total Organic Carbon / Total Nitrogen Analyser (TOC/TN). After a catalytic combustion, the nitrogen oxide was directed into a chemiluminiscence detector. Solutions of ammonium sulphate were used as standards.

\section{Results and Model development}

Since PHB is accumulated as an intracellular body and not excreted into the culture broth, if cells do not lyse, any change in biomass concentration can be attributed to either cellular growth, product formation or both. Cells act as catalysts for these two processes: creating new cellular matter $\left(x_{R}\right)$ and synthesizing the biopolymer $(\mathrm{P})$. Although ultimately linked, growth 
and PHB production can be considered as separate phenomena and, hence, the dynamics of each can be described by separate functions $\left(\mu_{x_{R}}, \mu_{P}\right)$. Thus, the first attempt in the current work was to model them independently, considering PHB production coupled negligibly to growth (i.e. PHB being non-growth associated). PHB degradation was not considered here knowing that in nitrogen free media with excess of carbon, the rate of degradation of PHB by hydrolysis is very low and does not affect the biopolymer content in the time frame used for fermentation [35]. Neither was cell death considered at his stage. The change with time for cell and product concentration is normally expressed through the specific growth rate and product rate respectively:

$$
\begin{aligned}
& \frac{d x_{R}}{d t}=\mu_{x_{R}} \cdot x_{R} \\
& \frac{d P H B}{d t}=\mu_{P} \cdot x_{R}
\end{aligned}
$$

In a closed system, such as a batch bioreactor, the rate of accumulation of products is equal to the generation rate of those as indicated in the mass balance equations 1 and 2. $x_{R}$ is often referred to as cell residue and was calculated as the difference between total biomass (CDM), measured gravimetrically, and PHB, measured in the GC-FID. Thus, $x_{R}$ is basically the cellular matter other than PHB and can be also expressed as non-PHB biomass. Together, $x_{R}$ and PHB, form total biomass. The classic Monod equation was used initially to describe specific growth rate:

$$
\mu=\frac{\mu_{m} \cdot S}{S+k_{S}}
$$

Given that the intention of the fermentations was producing PHB, carbon was always supplied in excess. Experiments varying the nitrogen concentration, with the concentration of other nutrients remaining constant, demonstrated that nitrogen was indeed the limiting substrate, controlling the rate and maximum amount of cells yielded. The non-PHB biomass at the end of the fermentation was found to be linearly related to the amount of nitrogen source initially present in the medium through a yield of $6.7 \mathrm{~g}$ non-PHB biomass/g nitrogen, which corresponds to the slope of the straight line in Fig. 1.

Fig.1 here

Tests with other nitrogen sources confirmed that cell growth could be improved by providing a greater availability of easily assimilable nitrogen forms, such as peptone. For the case of ammonium sulphate however, it has been observed that initial concentrations over $4 \mathrm{~g} / \mathrm{L}$ do not have a positive effect on growth. Heinzle and Lafferty [36] reported that the enzyme system using energy to assimilate $\mathrm{NH}_{4}^{+}$is repressed by high concentrations of ammonium cations. An inhibitory term was introduced into the equation to account for this phenomenon as follows:

$$
\mu_{x_{R}}=\frac{\mu_{m} \cdot N}{k_{N}+N} \cdot \frac{1}{1+\frac{N}{k_{I N}}}
$$

Where $\mu_{m}$ is the maximum specific growth rate, $N$ represents the nitrogen concentration in the media provided by the ammonium sulphate and $k_{N}, k_{I N}$ are respectively the half saturation constant in the Monod model and the inhibitory constant due to (large) nitrogen concentrations. 
At this stage, no threshold in terms of glycerol concentration that should be used for the fermentation is imposed. However, is likely that a large concentration of this carbon source will inhibit the rate at which cells proliferate, especially if they are not acclimatised to growing in glycerol. The rate equation was therefore extended to a double substrate model that can account for glycerol inhibition. This extended Monod equation can be written as follows:

$$
\mu_{x_{R}}=\frac{\mu_{m} \cdot N}{k_{N}+N} \cdot \frac{1}{1+\frac{N}{k_{I N}}} \cdot \frac{G l y}{G l y \cdot\left(1+\frac{G l y}{k_{I G}}\right)+k_{G}}
$$

The new terms added, $k_{G}$ and $k_{I G}$ correspond to the half saturation constant for glycerol (Gly) and the inhibitory constant of the new substrate incorporated.

The inhibitory terms chosen were selected from within those models that contain just one extra parameter to provide the best agreement with the experimental data. Four different equations, arising from the combinations of using Haldane's [37] and Andrews' [38] equations for each substrate (nitrogen and glycerol), were tested. As shown in Fig. 2, the extra term $\left(k_{S} \cdot S / k_{I}\right)$ found in the denominator of the Andrews equation causes a faster decrease in the specific growth rate (lower line). The two expressions derived from combining the Haldane and Andrews equations give similar results (middle lines) for the range of concentrations shown and fit better the experimental data. The expression finally chosen is that which contains the Andrews model for describing nitrogen inhibition and Haldane for glycerol, as it predicts a slightly slower decrease of the specific growth rate with increasing nitrogen. This is due to the lower impact that glycerol inhibition has in that combination.

$$
\mu=\frac{\mu_{m} \cdot S}{k_{N}+S+\frac{S^{2}}{k_{I}}} \quad \mu=\frac{\mu_{m} \cdot S}{\left(k_{N}+S\right) \cdot\left(1+\frac{S}{k_{I}}\right)}
$$

Fig. 2 here

As for growth, a modified Monod equation based on glycerol was also produced to describe the specific production rate. An excess of glycerol, the sole carbon source provided, promotes PHB production up to a certain level at which the system becomes "saturated". Large nitrogen concentrations promote acetyl-CoA, precursor of PHB, entering the TCA cycle and not going into the PHB synthesis pathway [39], and thus, a term for delaying the appearance of PHB production when nitrogen is still abundant in the media was included, making the expression analogous to the Haldane equation.

$$
\mu_{p}=\frac{\beta \cdot G l y}{G l y+k_{P}+\frac{N^{2}}{k_{I N P}}}
$$

$\beta$ can be defined as the non-growth associated specific rate constant for PHB production whereas $k_{P}$ and $k_{I P}$ are the half saturation constant for PHB production based on glycerol and the inhibition constant for PHB production due to nitrogen.

PHB production stops when the content inside the cell reaches around $80 \%$ of total dry biomass. Product inhibition was reported in the literature as the phenomenon causing the cessation of polymer formation [40]. Based on the Hill equation [41], an inhibitory term that reduces the growth rate by a factor less than 1 if PHB is present was introduced to describe what is believed to be the regulatory mechanism used by the cells. 
Where the integer exponent $n$ would be the equivalent to the Hill coefficient and $k_{d}$ the apparent dissociation constant.

Glycerol is consumed for product formation but also for the synthesis of nucleic acids and other cellular compounds via gluconeogenesis. Acetate, malate, formate or glucose are some of the intermediate metabolites that can be released when glycerol is used in the gluconeogenesis pathway according to Tanadchangsaeng and Yu [42]. However, no significant concentrations of organic acids were detected in the experiments conducted with glycerol concentrations below 40 g/L. Hence, glycerol consumption rate can be related directly to the rate of cell production and product formation through the yields of non-PHB biomass and PHB based on glycerol, $Y_{R} /_{G l y}$ and $Y_{P H B} /_{G l y}$ respectively, i.e.:

$$
-\frac{d G l y}{d t}=\left(\frac{1}{Y x_{R} / G l y} \cdot \mu_{x_{R}}+\frac{1}{Y_{P H B} / G l y} \cdot \mu_{p}\right) \cdot x_{R}
$$

Nitrogen consumption is computed by:

$$
-\frac{d N}{d t}=\frac{1}{Y x_{R} / N} \cdot \mu_{x_{R}} \cdot x_{R}
$$

Assuming nitrogen is only consumed for cell growth through $Y x_{R} /_{N}$, where $Y x_{R} /_{N}$ is the yield of non-PHB biomass based on nitrogen.

Several fermentations were carried out to establish a good reproducibility within the results. After that, a single experiment to provide definitive data used to estimate the model parameters was performed using $20 \mathrm{~g} / \mathrm{L}$ glycerol and $1 \mathrm{~g} / \mathrm{L}$ ammonium sulphate. To do this, a stochastic optimization based on a Simulated Annealing (SA) algorithm coupled with a deterministic method that uses the 'fmincon' function in MATLAB was used. The objective function for the optimisation was the minimum sum of squared errors between predicted and experimental values of the four state variables. A good fit to experimental data is achieved when low to medium glycerol concentrations are used. However, the model presented so far fails to describe accurately the values obtained when higher nitrogen concentrations are used, overpredicting the amount of PHB produced under those conditions, see Fig. 3.

Fig. 3 here

The simulation over predicts PHB production at higher initial nitrogen concentrations, since the theoretical PHB production does not stop until the carbon source is depleted. However, it is clear that in reality, other components, not accounted for in the model can become exhausted and therefore limit production.

PHB production rate is proportional to the amount of non-PHB biomass and, hence, the amount of PHB that can be formed is limited by the amount of cells. Several studies in the literature have suggested a self-inhibition mechanism that affects growth rate and this has been included in their equations for describing cell growth rate with different formulations, e.g. a cell density inhibition coefficient $[43,44]$. However, these models in addition to ours proposed above, still neglect the 
effects of PHB on cellular growth. It is only when growth and PHB production are expressed as functions of both compounds that a regulatory effect appears. This can be taken into account through the addition of a term with both variables included, which provides a factor between 0 and 1 to reduce the rates of both growth and PHB formation:

$$
\begin{gathered}
\frac{d x_{R}}{d t}=\frac{\mu_{m} N}{k_{N}+N} \cdot \frac{1}{1+\frac{N}{k_{I N}}} \cdot \frac{G l y}{G l y \cdot\left(1+\frac{G l y}{k_{I G}}\right)+k_{G}} \cdot x_{R} \cdot\left(1-\frac{x_{R}+P H B}{K_{x}}\right) \\
\frac{d P H B}{d t}=\frac{\beta \cdot G l y}{G l y+k_{P}+\frac{N^{2}}{k_{I N P}}} \cdot x_{R} \cdot\left(1-\frac{x_{R}+P H B}{K_{x}}\right)
\end{gathered}
$$

In this closed system, where nutrients are limited, cell growth and PHB formation compete for some of the same elements. Thus, both affect the rate at which the other occurs, creating a trade-off between cell proliferation and PHB formation. It is worth noting that no other inhibition term needs to be added to the differential equation for PHB, since its own concentration slows down the production rate. The extent to which this occurs is governed by a property of the system that can be defined as its carrying capacity. $K_{x}$.

$K_{x}$, is the upper threshold on growth size for a specific system under certain environmental conditions [43]. In this case, it is a function of the initial composition in the fermenter including inoculum concentration and operational factors such as agitation, which in turn influence dissolved oxygen. The carrying capacity represents the maximum biomass concentration that can be reached. The function of the term added to the differential equations 11 and 12 is to reduce the production rate as the system gets close to that upper bound. Although this parameter can be obtained from a given set of data, work needs to be done in order to estimate $K_{x}$ even when no experimental values are available, i.e. before conducting any fermentation.

For substrate consumption, equations must be modified accordingly:

$$
\begin{gathered}
-\frac{d G l y}{d t}=\left(\frac{1}{Y x_{R} / G l y} \cdot \mu_{x_{R}}+\frac{1}{Y_{P H B} / G l y} \cdot \mu_{p}\right) \cdot x_{R} \cdot\left(1-\frac{x_{R}+P H B}{K_{x}}\right) \\
-\frac{d N}{d t}=\frac{1}{Y x_{R / N}} \cdot \mu_{x_{R}} \cdot x_{R} \cdot\left(1-\frac{x_{R}+P H B}{K_{x}}\right)
\end{gathered}
$$

Where $\mu_{x_{R}}, \mu_{x_{p}}$ haven been previously defined in equations 5 and 7. The final model is formed by the four differential equations corresponding to non-PHB cell matter, PHB, glycerol and ammonium sulphate (11-14) containing 12 parameters, one less than in the previous version of the model. As previously described, the model parameters were estimated using experimental data corresponding to a representative fermentation conducted with $20 \mathrm{~g} / \mathrm{L}$ glycerol and $1 \mathrm{~g} / \mathrm{L}$ ammonium sulphate. Parameter values were estimated using the same methodology described earlier and, once obtained, they were treated as fixed constants for the C. necator-glycerol system. The results are shown in Table 1.

Table 1. here

The updated model is able to describe qualitative and quantitative biomass production and PHB formation, which indicates that an accurate distinction between cell biomass and intracellular product has been accomplished. The substrate consumptions can also be tracked throughout the fermentation with little deviation from the experimental data as seen in Fig. 4. It is important to note that the quality of the model predictions has been improved without increasing the degrees of freedom, i.e. number of parameters. 
Fig.4. here

\section{Model evaluation}

To test the robustness of the model and the applicability of the constants listed in Table 1, simulations of fermentations carried out with different amounts of carbon and nitrogen in the medium were run. Results are contrasted to experimental data and presented in Fig.5, 6.

Fig.5. here

The reliability of the model is maintained for different conditions including changes in the nitrogen and carbon source. The success in forecasting the final concentrations as well as the patterns with time indicates that a reasonable distinction between substrates being converted into one product and the other has been achieved. Whenever glycerol is increased and nitrogen kept constant, an increase in PHB production is seen, both in theoretical and empirical results. Non-PHB biomass remains constant as it is mainly governed by the nitrogen source. On the other hand, as the supply of nitrogen is increased but glycerol is maintained constant, non-PHB biomass rises to a maximum level and PHB concentration suffers a significant decrease. Hence, the content of PHB in the cells diminishes. Through this formulation, production may stop, even if where there is still glycerol present in the media, and the product concentrations tend to a constant value by the end of the fermentation. The model estimates the fermentation outcome to within $\pm 10 \%$ of the measured values.

Fig.6. here

The kinetic constants shown here (Table 1) are applicable to PHB formation by C. necator when using glycerol as sole carbon nutrient and ammoniumn sulphate as nitrogen source. They were maintained as constant for all the simulations performed to test the model performance and degree of fulfillment under different conditons where the biopolymer can be synthesized. The maximum specific growth rate reported is higher than the one observed from experimental data (specific growth rate during the exponential phase). This is due to the fact that the Monod equation was modified to allow for a double substrate type of model with inhibition. The inhibition constants, $k_{I N}$ and $k_{I G}$ can give an idea of the maximum concentrations to be used before those compounds start becoming inhibitory. In our case, PHB accumulation started when nitrogen was almost depleted, which explains the low value of $k_{I N P}$. The yields of non-PHB biomass cells and PHB are close to the values we would obtain when applying a mass balance to glycerol and the stoichiometric coefficients found in literature for cell and PHB production from glycerol [44, 45]. The carrying capacity, matches the overall concentration obtained experimentally but it is accepted that it would need to be increased when scaling up the process. It is possible to predict the steady state concentration of non-PHB biomass using the given yield of non-PHB biomass cells based on nitrogen. However, nitrogen consumption occurs faster than the model predicts, suggesting that this nutrient is used for purposes other than growth, e.g. cell maintenance. An additional term could be added to the model to account for this but would introduce additional degrees of freedom that could not easily be distinguished from experimental data without significant further experimentation, specifically designed to separate the effects of maintenance and growth. 
The model was also used predictively in order to evaluate the effect of carbon and nitrogen over a wider range of situations than those which could be practically conducted. Maximum product concentration and specific growth rate were two of the variables considered as having a critical impact in the fermentation feasability. Fig. 7 shows the maximum concentration of PHB that can be achieved (in our laboratory system) as a function of the two main media components. It is easy to identify those combinations of concentration which promote a higher production and those that do the opposite. The proposed competition effect becomes clear when looking at that region of very large nitrogen concenteration. Also, it is important to emphasize that excess glycerol above $60 \mathrm{~g} / \mathrm{L}$ does not lead to higher PHB accumation and should be avoided in order not to have unused substrate in the media or undesirable secondary products.

Fig. 7 here

Specific growth rate is a key factor to ensure good productivities in batch fermenters but also of utmost importance in continuous fermentations where it determines the maximum dilution rate that can be used without risking biomass wash-out. The heterologous substrate concept assumes that growth rate can be affected simultaneously by more than one substrate, exactly as happens for this system [46] and this explains the interest in studying them simultaneously. The initial conditions that should be used to ensure a high specific growth rate or those substrate and nutrient concentrations to be maintained in the reactor in a flow system can be readily predicted through $3 \mathrm{D}$ plots, such as the one shown in Fig. 8.

Fig. 8 here

\section{Conclusions}

A new model using a modified Monod equation for cell and product formation was created based on a mechanistic understanding of the major phenomena observed experimentally. Carbon and nitrogen were the two main factors of study, given their impact in PHB production. Using a single, representative set of model parameters (constants), simulations under different conditions from those used to generate them were successfully carried out. Despite the simplicity of the model and the use of just a single set of parameters, good differentiation in substrate consumption and conversion to different products was obtained. Realistic predictions, when larger nutrient concentrations are used, were obtained by setting a threshold in terms of maximum biomass formation. This confirms that the approach of adopting only the major influencing factors in the model can still generate acceptable outputs. The applicability of the model has been shown for tasks such as selecting the initial or operational conditions to get optimal performance (highest PHB concentration, highest specific growth rate). As the product of interest, PHB, is intracellular, increased growth rate will improve the productivity and a higher PHB content will reduce separation costs. The interaction between carbon and nitrogen components and their effect on those fermentation parameters was clearly demonstrated. Within the process, important kinetic parameters have been determined that can be widely used when planning the process design. The importance of the approach adopted here is that, the model relies on fixed constants and not parameters which are tuned with every set of experiments. This means that experimental data is not necessary for running simulations but, instead, simulations can replace experiments. The purpose of the model, to become a predictive tool for process development, will be extended to assist the selection of the mode of operation in the near future. 


\section{Acknowledgements}

The authors wish to thank the University of Manchester for the provision of a President's Doctoral Scholarship Award to Cristina Pérez Rivero.

\section{References}

[1] R.A.J. Verlinden, D.J. Hill, M.A. Kenward, C.D. Williams, I. Radecka, Bacterial synthesis of biodegradable polyhydroxyalkanoates, Journal of Applied Microbiology, 102 (2007) 1437-1449.

[2] M. Tolinski, Plastics and sustainability: towards a peaceful coexistence between bio-based and fossil fuel-based plastics, John Wiley \& Sons, 2011.

[3] Z. Hanley, T. Slabas, K.M. Elborough, The use of plant biotechnology for the production of biodegradable plastics, Trends in Plant Science, 5 (2000) 45-46.

[4] G.-Y.A. Tan, C.-L. Chen, L. Li, L. Ge, L. Wang, I.M.N. Razaad, Y. Li, L. Zhao, Y. Mo, J.-Y. Wang, Start a research on biopolymer polyhydroxyalkanoate (PHA): a review, Polymers, 6 (2014) 706-754.

[5] C. Du, J. Sabirova, W. Soetaert, S. Ki Carol Lin, Polyhydroxyalkanoates production from low-cost sustainable raw materials, Current Chemical Biology, 6 (2012) 14-25.

[6] K. Tanaka, A. Ishizaki, T. Kanamaru, T. Kawano, Production of Poly (D-3-Hydroxybutyrate) from $\mathrm{CO}_{2}, \mathrm{H}_{2}$, and $\mathrm{O}_{2}$ by High Cell Density Autotrophic Cultivation of Alcaligenes eutrophus, Biotechnology and Bioengineering, 45 (1995) 268-275.

[7] L.S. Serafim, P.C. Lemos, M.G. Albuquerque, M.A. Reis, Strategies for PHA production by mixed cultures and renewable waste materials, Applied Microbiology and Biotechnology, 81 (2008) 615-628.

[8] W.S. Ahn, S.J. Park, S.Y. Lee, Production of poly (3-hydroxybutyrate) from whey by cell recycle fed-batch culture of recombinant Escherichia coli, Biotechnology Letters, 23 (2001) 235-240.

[9] C. Zhang, Biodegradable Polyesters: Synthesis, Properties, Applications, in: Biodegradable Polyesters, Wiley-VCH Verlag GmbH \& Co. KGaA, (2015), pp. 1-24.

[10] J.M. Cavalheiro, M.C.M. de Almeida, C. Grandfils, M. Da Fonseca, Poly (3-hydroxybutyrate) production by Cupriavidus necator using waste glycerol, Process Biochemistry, 44 (2009) 509-515.

[11] F. Yang, M.A. Hanna, R. Sun, Value-added uses for crude glycerol-a byproduct of biodiesel production, Biotechnol Biofuels, 5 (2012) 1-10.

[12] G. Mothes, C. Schnorpfeil, J.U. Ackermann, Production of PHB from Crude Glycerol, Engineering in Life Sciences, 7 (2007) 475-479.

[13] A. Salakkam, C. Webb, The inhibition effect of methanol, as a component of crude glycerol, on the growth rate of Cupriavidus necator and other micro-organisms, Biochemical Engineering Journal, 98 (2015) 84-90.

[14] E.A. Dawes, P.J. Senior, The role and regulation of energy reserve polymers in micro-organisms, Advances in microbial physiology, 10 (1972) 135-266.

[15] A.J. Anderson, E.A. Dawes, Occurrence, metabolism, metabolic role, and industrial uses of bacterial polyhydroxyalkanoates, Microbiological reviews, 54 (1990) 450-472.

[16] P. Chakraborty, W. Gibbons, K. Muthukumarappan, Conversion of volatile fatty acids into polyhydroxyalkanoate by Ralstonia eutropha, Journal of Applied Microbiology, 106 (2009) 1996-2005.

[17] M. Zinn, B. Witholt, T. Egli, Dual nutrient limited growth: models, experimental observations, and applications, Journal of Biotechnology, 113 (2004) 263-279.

[18] M. Novak, M. Koller, M. Braunegg, P. Horvat, Mathematical modelling as a tool for optimized PHA production, Chemical and Biochemical Engineering Quarterly, 29 (2015) 183-220.

[19] A. Mulchandani, J. Luong, C. Groom, Substrate inhibition kinetics for microbial growth and synthesis of poly- $\beta$ hydroxybutyric acid by Alcaligenes eutrophus ATCC 17697, Applied Microbiology and Biotechnology, 30 (1989) 11-17.

[20] P. Patwardhan, A. Srivastava, Model-based fed-batch cultivation of $R$. eutropha for enhanced biopolymer production, Biochemical Engineering Journal, 20 (2004) 21-28.

[21] J. Luong, Generalization of Monod kinetics for analysis of growth data with substrate inhibition, Biotechnology and Bioengineering, 29 (1987) 242-248.

[22] R. Dhanasekar, T. Viruthagiri, P.L. Sabarathinam, Poly(3-hydroxy butyrate) synthesis from a mutant strain Azotobacter vinelandii utilizing glucose in a batch reactor, Biochemical Engineering Journal, 16 (2003) 1-8.

[23] T. Katoh, D. Yuguchi, H. Yoshii, H. Shi, K. Shimizu, Dynamics and modeling on fermentative production of poly ( $\beta$ hydroxybutyric acid) from sugars via lactate by a mixed culture of Lactobacillus delbrueckii and Alcaligenes eutrophus, Journal of Biotechnology, 67 (1999) 113-134.

[24] J.H. Lee, H.C. Lim, J. Hong, Application of nonsingular transformation to on-line optimal control of poly- $\beta$ hydroxybutyrate fermentation, Journal of Biotechnology, 55 (1997) 135-150.

[25] M. Tohyama, T. Patarinska, Z. Qiang, K. Shimizu, Modeling of the mixed culture and periodic control for PHB production, Biochemical Engineering Journal, 10 (2002) 157-173.

[26] I.V. Spoljarić, M. Lopar, M. Koller, A. Muhr, A. Salerno, A. Reiterer, K. Malli, H. Angerer, K. Strohmeier, S. Schober, Mathematical modeling of poly [(R)-3-hydroxyalkanoate] synthesis by Cupriavidus necator DSM 545 on substrates stemming from biodiesel production, Bioresource technology, 133 (2013) 482-494. 
[27] I.V. Špoljarić, M. Lopar, M. Koller, A. Muhr, A. Salerno, A. Reiterer, P. Horvat, In silico optimization and low structured kinetic model of poly[(R)-3-hydroxybutyrate] synthesis by Cupriavidus necator DSM 545 by fed-batch cultivation on glycerol, Journal of Biotechnology, 168 (2013) 625-635.

[28] W.S. Kim, Cybernetic model for synthesis of poly- $\beta$-hydroxybutyric acid in Alcaligenes eutrophus, Biotechnology and Bioengineering, 43 (1994) 1043-1051.

[29] M. Lopar, I.V. Špoljarić, N. Cepanec, M. Koller, G. Braunegg, P. Horvat, Study of metabolic network of Cupriavidus necator DSM 545 growing on glycerol by applying elementary flux modes and yield space analysis, Journal of industrial microbiology \& biotechnology, 41 (2014) 913-930.

[30] K.G. Gadkar, J.D. Francis III, T.J. Crowley, J.D. Varner, Cybernetic model predictive control of a continuous bioreactor with cell recycle, Biotechnology progress, 19 (2003) 1487-1497.

[31] P. Patnaik, Neural network designs for poly- $\beta$-hydroxybutyrate production optimization under simulated industrial conditions, Biotechnology Letters, 27 (2005) 409-415.

[32] P.R. Patnaik, Design considerations in hybrid neural optimization of fed-batch fermentation for PHB production by Ralstonia eutropha, Food and Bioprocess Technology, 3 (2010) 213-225.

[33] B.S. Kim, S.Y. Lee, H.N. Chang, Production of poly- $\beta$-hydroxybutyrate by fed-batch culture of recombinant Escherichia coli, Biotechnology Letters, 14 (1992) 811-816.

[34] V. Riis, W. Mai, Gas chromatographic determination of poly- $\beta$-hydroxybutyric acid in microbial biomass after hydrochloric acid propanolysis, Journal of Chromatography A, 445 (1988) 285-289.

[35] P.R. Patnaik, Perspectives in the modeling and optimization of PHB production by pure and mixed cultures, Critical reviews in biotechnology, 25 (2005) 153-171.

[36] E. Heinzle, R.M. Lafferty, A kinetic model for growth and synthesis of poly- $\beta$-hydroxybutyric acid (PHB) in Alcaligenes eutrophus H 16, European J. Appl. Microbiol. Biotechnol., 11 (1980) 8-16.

[37] J. Haldane, s.(1930) Enzymes, in, Longmans, London.

[38] J.F. Andrews, Dynamic model of the anaerobic digestion process, Journal of the Sanitary Engineering Division, 95 (1969) 95-116.

[39] S. Gaspard, M.C. Ncibi, Biomass for Sustainable Applications: Pollution Remediation and Energy, Royal Society of Chemistry, 2013.

[40] P. Raje, A.K. Srivastava, Updated mathematical model and fed-batch strategies for poly- $\beta$-hydroxybutyrate (PHB) production by Alcaligenes eutrophus, Bioresource technology, 64 (1998) 185-192.

[41] A.V. Hill, The possible effects of the aggregation of the molecules of haemoglobin on its dissociation curves, J Physiol (Lond), 40 (1910) 4-7.

[42] N. Tanadchangsaeng, J. Yu, Microbial synthesis of polyhydroxybutyrate from glycerol: gluconeogenesis, molecular weight and material properties of biopolyester, Biotechnology and Bioengineering, 109 (2012) 2808-2818.

[43] A. Tsoularis, J. Wallace, Analysis of logistic growth models, Mathematical biosciences, 179 (2002) 21-55.

[44] M.S.I. Mozumder, L. Goormachtigh, L. Garcia-Gonzalez, H. De Wever, E.I. Volcke, Modeling pure culture heterotrophic production of polyhydroxybutyrate (PHB), Bioresource technology, 155 (2014) 272-280.

[45] M. Akiyama, T. Tsuge, Y. Doi, Environmental life cycle comparison of polyhydroxyalkanoates produced from renewable carbon resources by bacterial fermentation, Polymer Degradation and Stability, 80 (2003) 183-194.

[46] G. Okpokwasili, C. Nweke, Microbial growth and substrate utilization kinetics, African Journal of Biotechnology , 5 (2006) 305-317. 


\section{Figure 1}

Figure 1.

Relationship between non-PHB biomass produced during 100 hours of fermentation and the initial nitrogen concentration in the medium for batch flask experiments.

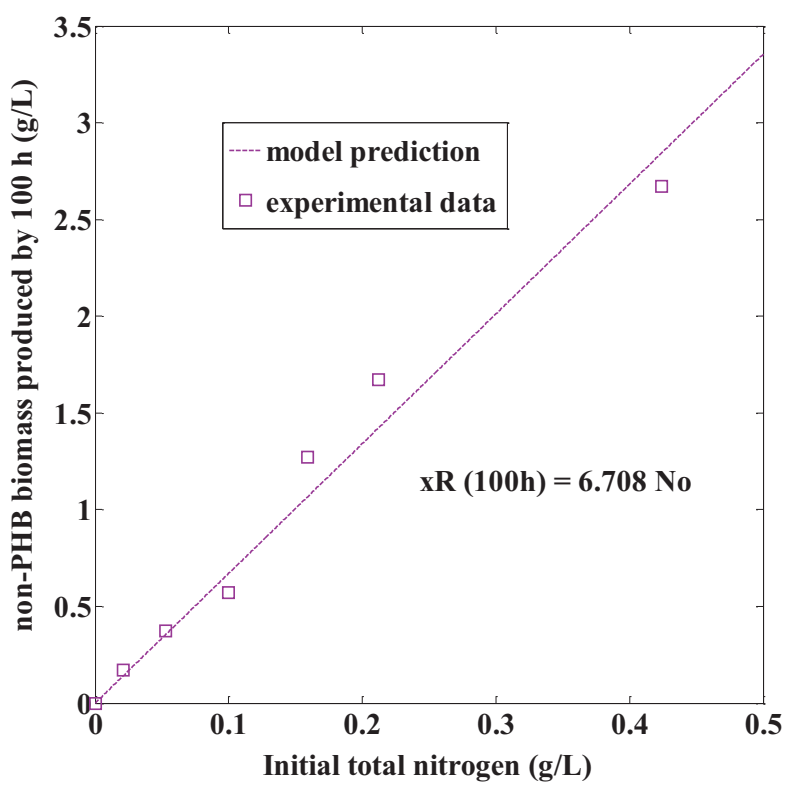


Figure 2.

Experimental values of specific growth rate for fermentations conducted with $30 \mathrm{~g} / \mathrm{L}$ glycerol and model predictions as a function of the initial ammonium sulphate concentration according to different equations for substrate inhibition: $\mu_{m}=0.35 h^{-1}, k_{N}=0.125 \mathrm{~g} / \mathrm{L}, k_{I N}=1.25 \mathrm{~g} / \mathrm{L}, k_{G}=$ $10 \mathrm{~g} / \mathrm{L}, k_{I G}=100 \mathrm{~g} / \mathrm{L}$.

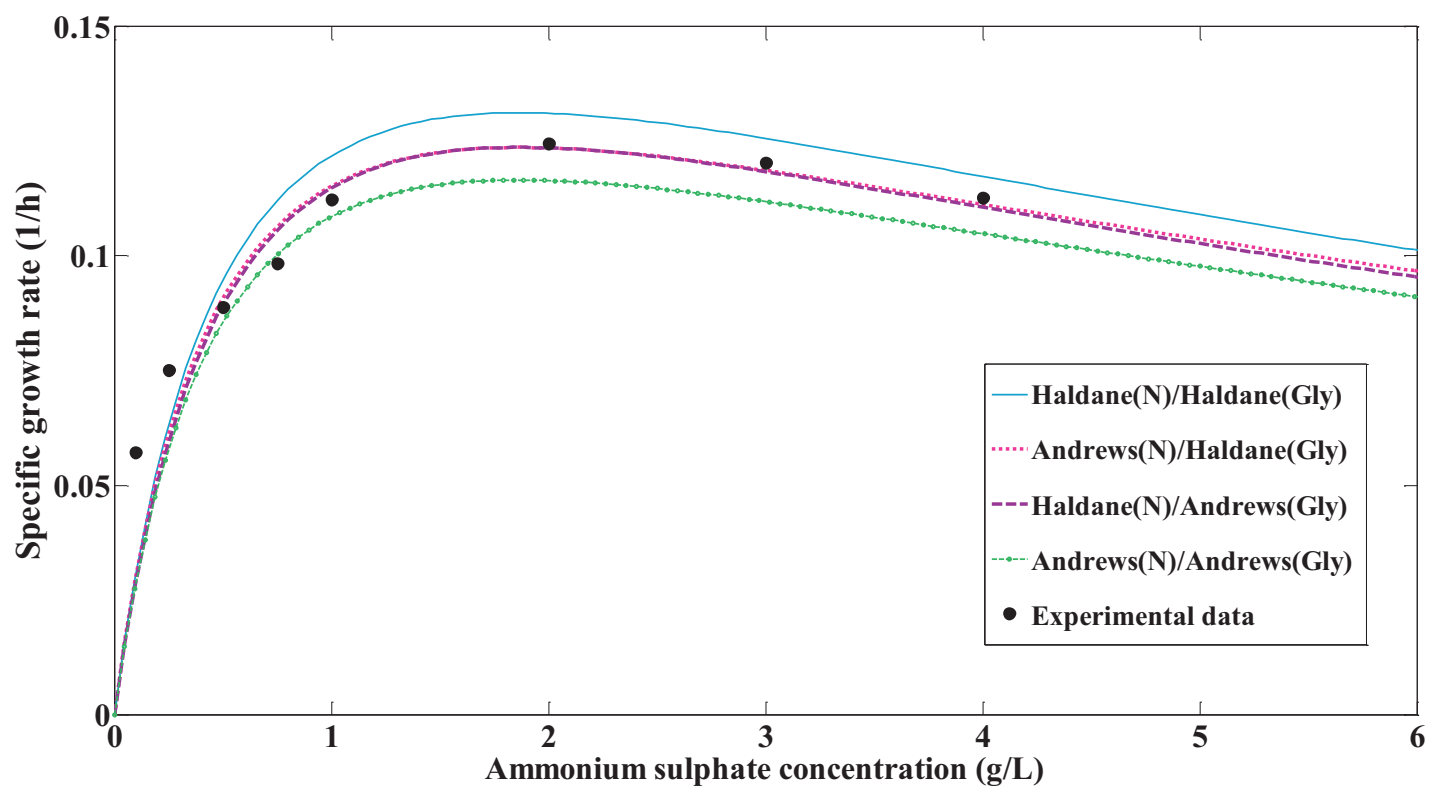




\section{Figure 3.}

Experimental data and model predictions for fermentations conducted under different conditions: 20 $\mathrm{g} / \mathrm{L}$ glycerol, $1 \mathrm{~g} / \mathrm{L}$ ammonium sulphate (top left), $30 \mathrm{~g} / \mathrm{L}$ glycerol, $1 \mathrm{~g} / \mathrm{L}$ ammonium sulphate (top right), $30 \mathrm{~g} / \mathrm{L}$ glycerol, $2 \mathrm{~g} / \mathrm{L}$ ammonium sulphate (bottom left), $30 \mathrm{~g} / \mathrm{L}$ glycerol, $3 \mathrm{~g} / \mathrm{L}$ ammonium sulphate (bottom right).
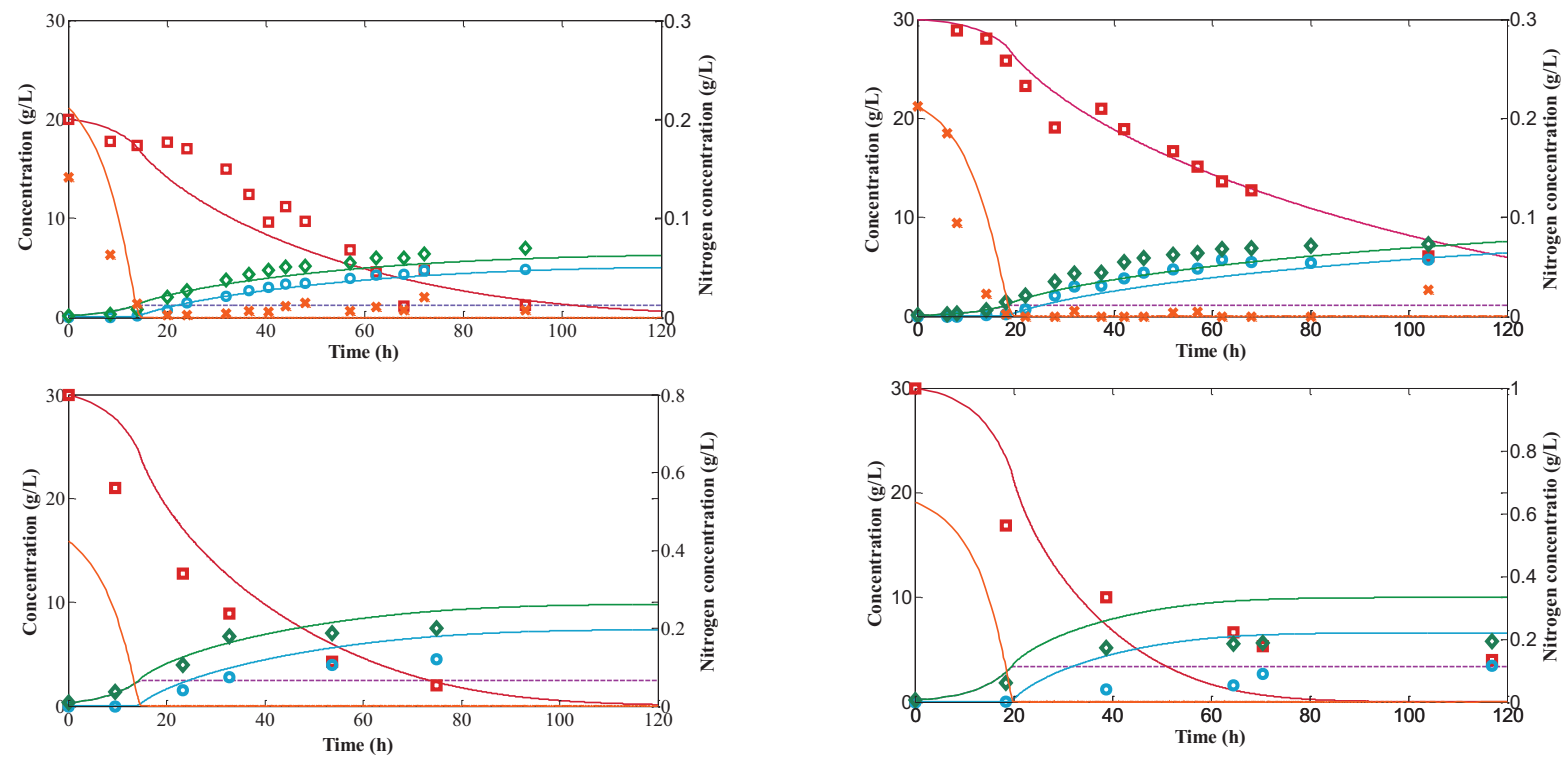

- Gly prediction $\square$ Gly data - PHB prediction $\triangle \mathrm{PHB}$ data ${ }^{--}$- $R$ prediction - Total biomass prediction $\triangle \mathrm{CDM}$ data $-\mathrm{N}$ prediction $\times \mathrm{N}$ data 
Figure 4.

Experimental results (symbols) and model predictions (lines) for the profiles of total biomass, glycerol, PHB and nitrogen concentration over time using $20 \mathrm{~g} / \mathrm{L}$ glycerol and $1 \mathrm{~g} / \mathrm{L}$ ammonium sulphate in batch mode cultivation.
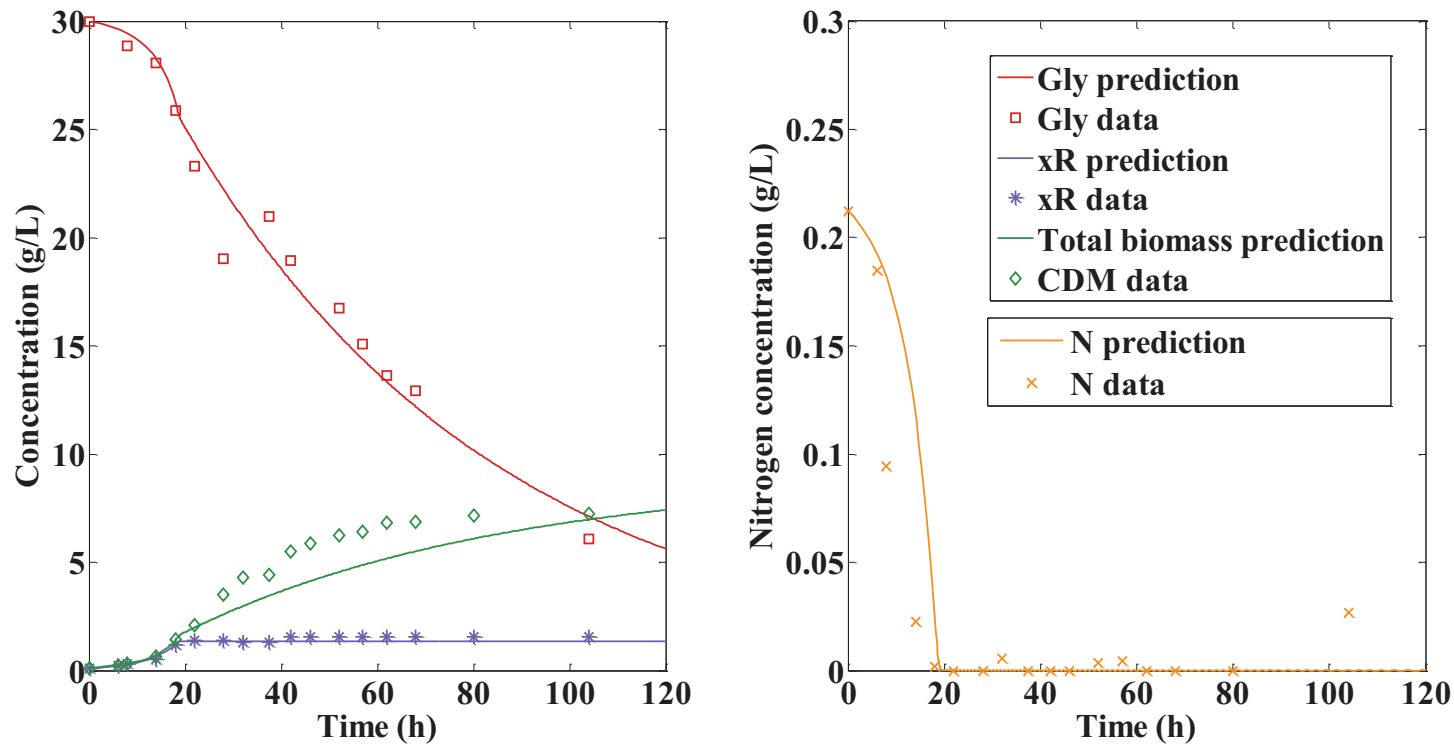


\section{Figure 5.}

Experimental results (symbols) and model predictions (lines) for the profiles of total biomass, glycerol, PHB and nitrogen concentration over time using $30 \mathrm{~g} / \mathrm{L}$ glycerol and $1 \mathrm{~g} / \mathrm{L}$ ammonium sulphate in batch mode cultivation.
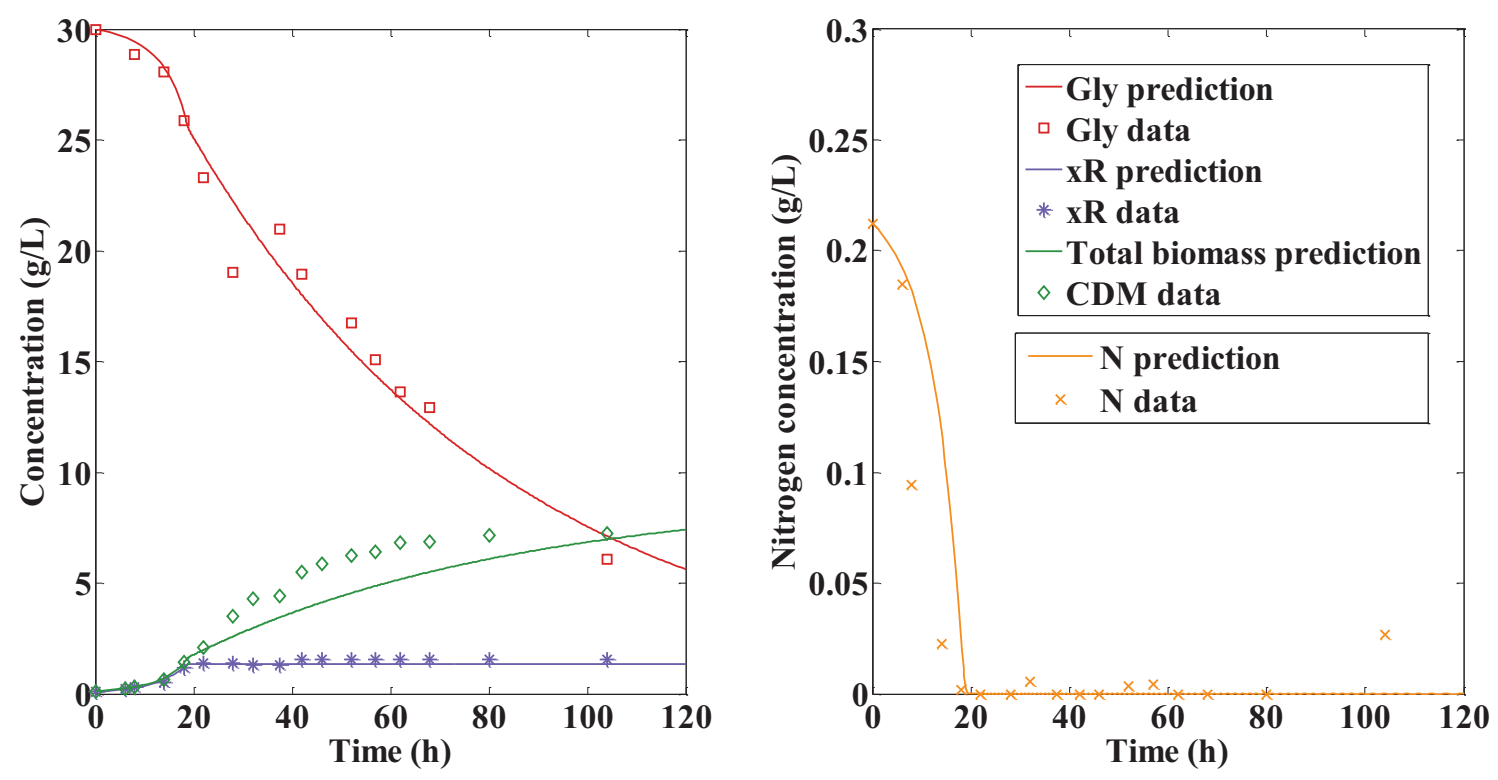


\section{Figure 6.}

Relationship between PHB, total biomass and non-PHB biomass concentration produced by the end of the fermentation and initial ammonium sulphate concentration. The initial glycerol concentration was $30 \mathrm{~g} / \mathrm{L}$ of glycerol in every case.
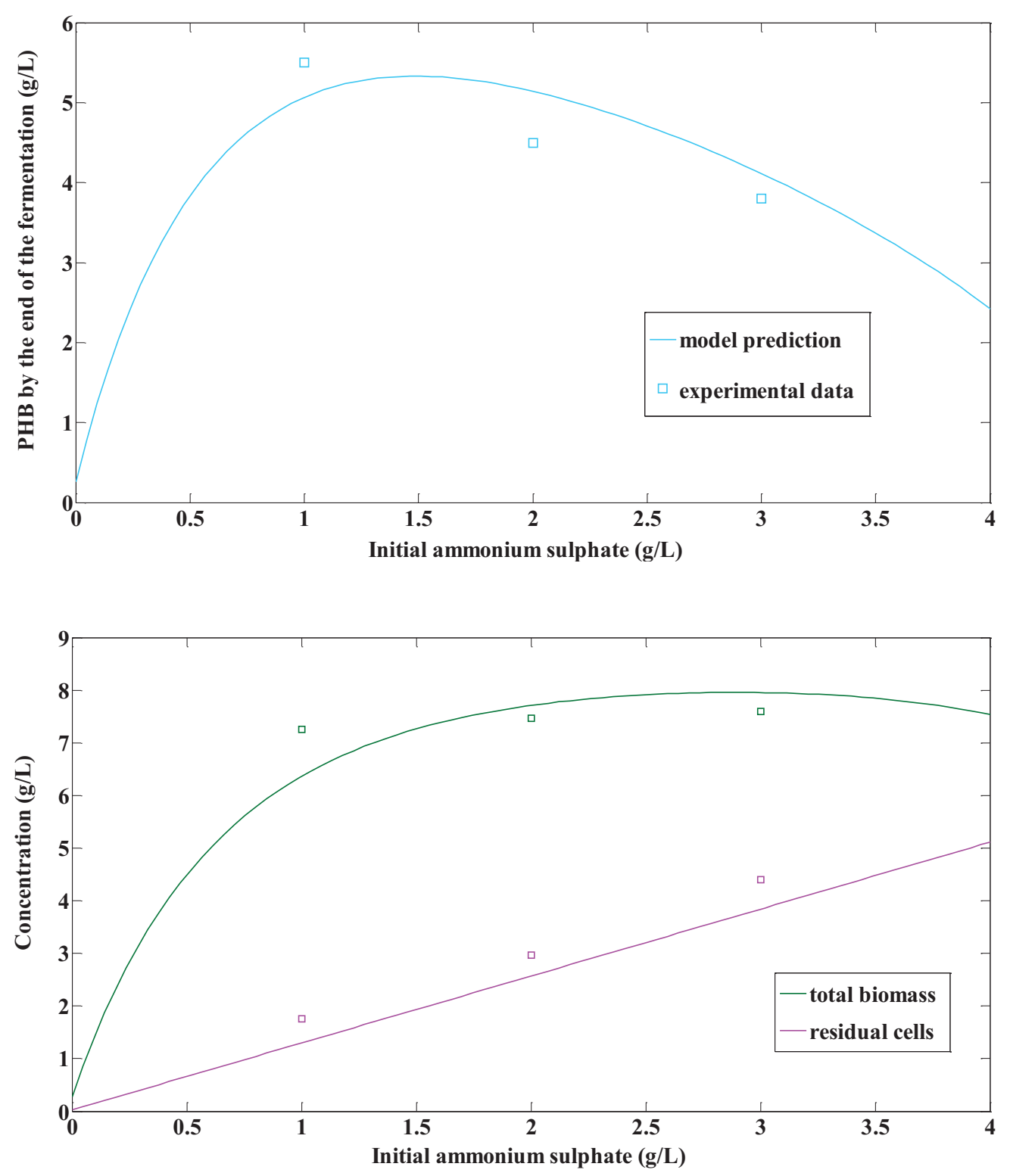


\section{Figure 7}

\section{Figure 7}

Contour plot of the maximum PHB concentration achievable as a function of the initial nitrogen and carbon concentrations, predicted using the model presented above

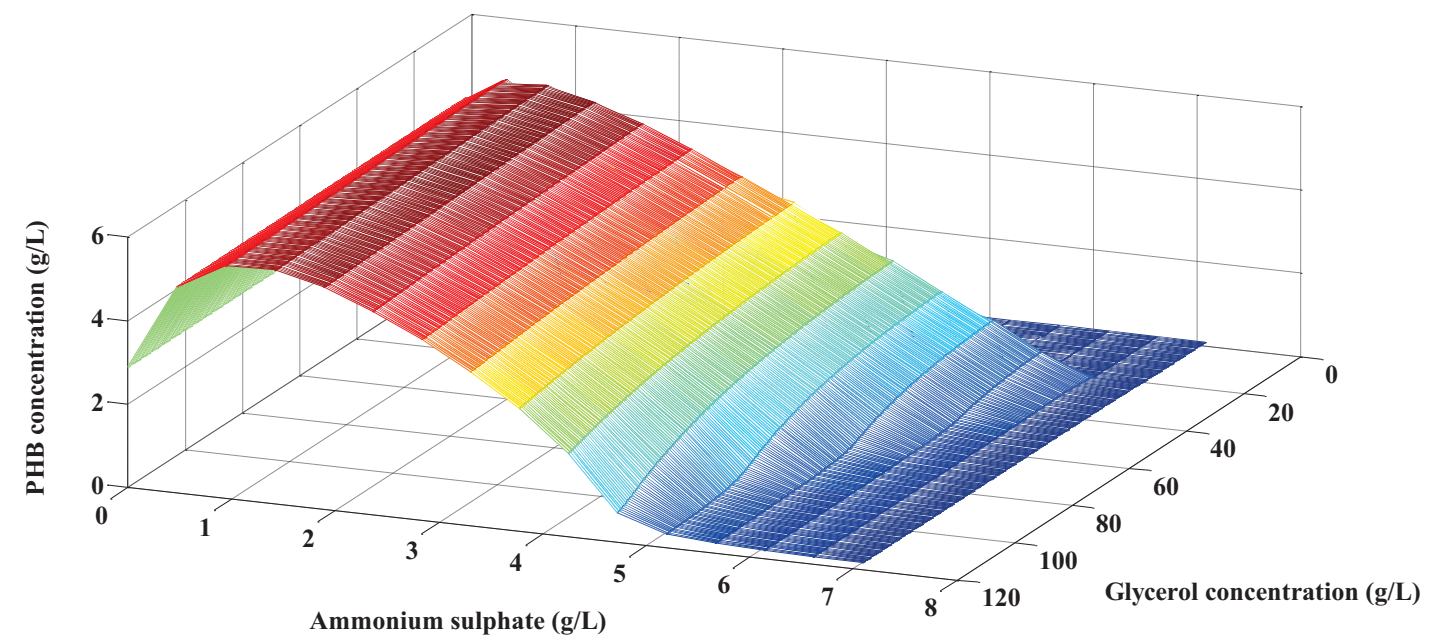




\section{Figure 8}

\section{Figure 8}

Contour plot of predicted specific growth rate for $C$. necator as a function of the nitrogen and carbon concentrations in the fermentation broth

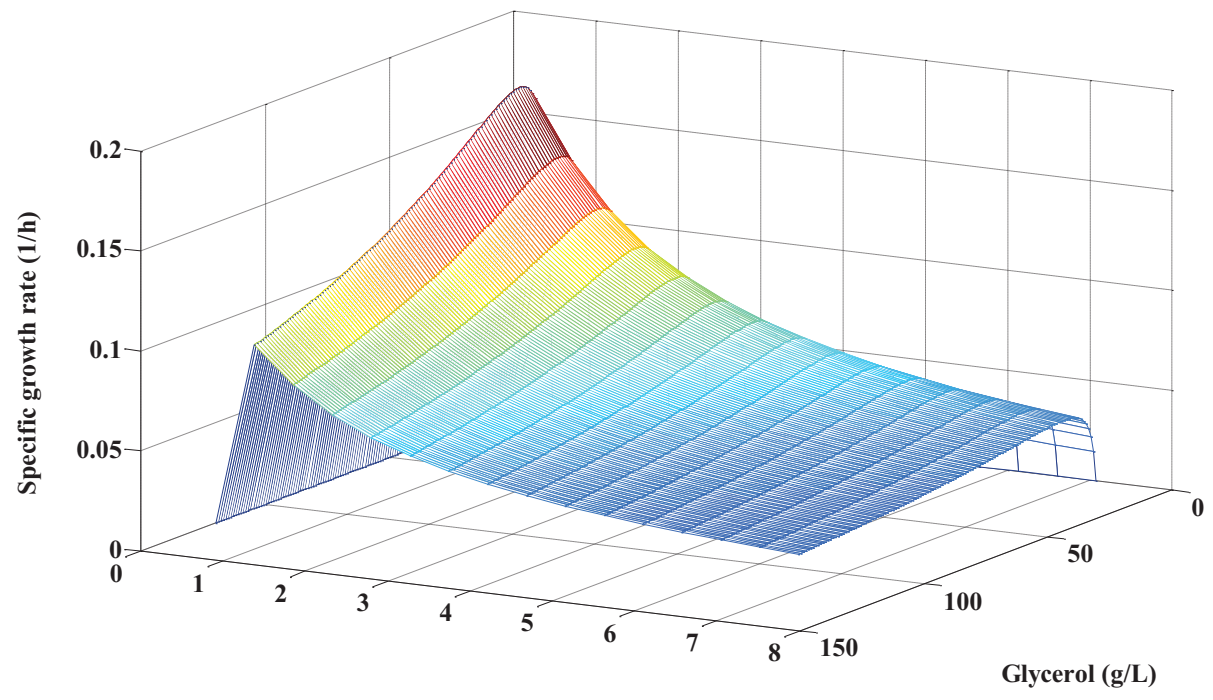

Ammonium sulphate (g/L) 


\section{Table 1.}

Computed parameters for the two low-structured models presented for describing PHB synthesis from glycerol by $C$. necator. Results were based on a fermentation conducted with $20 \mathrm{~g} / \mathrm{L}$ glycerol and 1 $\mathrm{g} / \mathrm{L}$ ammonium sulphate.

\begin{tabular}{|c|c|c|}
\hline Parameter & Preliminary Model Value & Updated Model Value \\
\hline$\mu_{m}$ & $0.20 h^{-1}$ & $0.35 h^{-1}$ \\
\hline $\boldsymbol{k}_{N}$ & $0.04 \mathrm{~g} / \mathrm{L}$ & $0.01 \mathrm{~g} / \mathrm{L}$ \\
\hline$k_{I N}$ & $0.50 \mathrm{~g} / \mathrm{L}$ & $0.27 \mathrm{~g} / \mathrm{L}$ \\
\hline$k_{I G}$ & $74.00 \mathrm{~g} / \mathrm{L}$ & $86.31 \mathrm{~g} / \mathrm{L}$ \\
\hline $\boldsymbol{k}_{G}$ & $0.60 \mathrm{~g} / \mathrm{L}$ & $1.05 \mathrm{~g} / \mathrm{L}$ \\
\hline $\boldsymbol{K}_{x}$ & - & $9.00 \mathrm{~g} / \mathrm{L}$ \\
\hline$n$ & 1.00 & - \\
\hline$\beta$ & 0.14 & $0.10 h^{-1}$ \\
\hline $\boldsymbol{k}_{p}$ & 1.60 & $0.01 \mathrm{~g} / \mathrm{L}$ \\
\hline$k_{I N P}$ & $5 \cdot 10^{-4}(g / L)^{2}$ & $1 \cdot 10^{-3}(g / L)^{2}$ \\
\hline$Y x_{R} / G L Y$ & 0.39 & $0.35 \frac{\text { g non PHB biomass }}{g \text { glycerol }}$ \\
\hline$Y_{P H B} / G L Y$ & 0.34 & $029 \frac{\text { g PHB biomass }}{\text { g glycerol }}$ \\
\hline$Y x_{R / N}$ & 6.30 & $6.0 \frac{\text { g non PHB biomass }}{g \text { glycerol }}$ \\
\hline$k_{d}$ & $9.00 \mathrm{~g} / \mathrm{L}$ & - \\
\hline
\end{tabular}

